05

\title{
Применение модели делокализованных атомов к металлическим стеклам
}

\author{
(ㄷ Д.С. Сандитов, ${ }^{1,2}$ М.В. Дармаев, ${ }^{1}$ Б.Д. Сандитов ${ }^{1}$ \\ ${ }^{1}$ Бурятский государственный университет, \\ 670000 Улан-Удэ, Россия \\ ${ }^{2}$ Институт фризического материаловедения СО РАН, \\ 670047 Улан-Удэ, Россия \\ e-mail: sanditov@bsu.ru
}

(Поступило в Редакцию 15 марта 2016 г)

По данным об эмпирических постоянных уравнения Фогеля-Фульчера-Таммана (для температурной зависимости вязкости) проведен расчет параметров модели делокализованных атомов применительно к металлическим стеклам. Показано, что для них справедлив тот же критерий стеклования, что и у аморфных органических полимеров и неорганических стекол. Этот факт подтверждает в качественном отношении универсальность основных закономерностей процесса перехода жидкость-стекло для всех аморфных веществ независимо от их природы. Энергия делокализации атома в металлических стеклах $\Delta \varepsilon_{e} \approx 20-25 \mathrm{~kJ} / \mathrm{mol}$ совпадает с данными для оксидных неорганических стекол. Она существенно ниже энергий активации вязкого течения и процесса диффузии ионов. Делокализация атома - его смещение из равновесного положения - для аморфных металлических сплавов представляет собой низкоэнергетический мелкомасштабный процесс, как и у других стеклообразных систем.

DOI: 10.21883/JTF.2017.01.44017.1807

\section{Введение. Модель делокализованных атомов}

Настоящая работа посвящена расчету параметров модели делокализованных атомов [1] по данным о вязкости для металлических стекол (аморфных металлических сплавов).

Необходимым условием делокализации активного (возбужденного) атома - его смещения из равновесного положения - является образование вблизи него элементарного объема $\Delta v_{e}$ в результате локальных тепловых флуктуаций плотности $[1,2]$. Если умножим $\Delta v_{e}$ на число делокализованных атомов $N_{e}$, получим флуктуационный объем аморфной среды $\Delta V_{e}$

$$
\Delta V_{e}=N_{e} \Delta v_{e}
$$

Кинетические единицы аморфных веществ отличаются друг от друга, главным образом, величиной флуктуационного объема $\tilde{v}$, который образуется в их окрестности. Число частиц $d n$, обладающих флуктуационным объемом от $\tilde{v}$ до $\tilde{v}+d \tilde{v}$, описывается функцией $[1,3]$

$$
d n=A \exp \left(-\frac{\Delta H}{k T}\right) d \tilde{v}=A \exp \left[-\frac{\left(p_{i}+p\right) \tilde{v}}{k T}\right] d \tilde{v},
$$

где $\Delta H=\left(p_{i}+p\right) \tilde{v}$ - энтальпия образования объема $\tilde{v}$. Сумма внутреннего $p_{i}$ и внешнего $p$ давлений $\left(p_{i}+p\right)$ выступает в качестве константы, не зависящей от $\tilde{v}$. После нормировки

$$
\int_{0}^{N} d n=N
$$

позволяющей получить соответствующее выражение $(N-$ число атомов $)$

$$
A=\frac{\left(p_{i}+p\right) N}{k T}
$$

находим число делокализованных атомов $N_{e}$, у которых $\tilde{v} \geq \Delta v_{e}$

$$
\begin{aligned}
N_{e} & =\int_{\Delta v_{e}}^{\infty} \frac{\left(p_{i}+p\right) N}{k T} \exp \left[-\frac{\left(p_{i}+p\right) \tilde{v}}{k T}\right] d \tilde{v} \\
& =N \exp \left[-\frac{\left(p_{i}+p\right) \Delta v_{e}}{k T}\right]
\end{aligned}
$$

Определив флуктуационный объем аморфного вещества $\Delta V_{e}$ как сумму элементарных объемов

$$
\Delta V_{e}=\int_{0}^{N} \tilde{v} d n=\frac{N k T}{p_{i}+p}
$$

для среднего флуктуационного объема $v_{f}=\Delta V_{e} / N$, приходящегося на частицу, можно получить соотношение

$$
v_{f}=\frac{k T}{\left(p_{i}+p\right)} .
$$

Подстановка данного выражения в равенство (1) приводит к формуле вероятности делокализации атома $W_{e}=N_{e} / N$

$$
W_{e}=\exp \left(-\frac{\Delta v_{e}}{v_{f}}\right)
$$




\section{Вязкость и флуктуационный объем в области стеклования жидкостей}

Используя соотношение (2) и известную связь между вязкостью $\eta$ и величиной $W_{e}$ в соответствии с теорией Стокса-Эйнштейна: $\eta \sim 1 / W_{e}$, приходим к следующему уравнению вязкости [1]:

$$
\eta=\eta_{0} \exp \left(\frac{\Delta v_{e}}{v_{f}}\right) \cong \eta_{0} \exp \left(\frac{1}{f}\right),
$$

где $\eta_{0}-$ коэффициент пропорциональности (вязкость $\eta$ при $T \rightarrow \infty), f=\left(\Delta V_{e} / V\right)$ - доля флуктуационного объема. Поскольку элементарный объем $\Delta v_{e}$ близок к атомному объему $v=V / N$, мы здесь приняли, что $f=v_{f} / \Delta v_{e} \approx v_{f} / v$.

Приняв в уравнении вязкости (3) линейную аппроксимацию температурной зависимости доли флуктуационного объема $f(T)$ вблизи температуры стеклования $T_{g}$

$$
f=f_{g}+\beta_{f}\left(T-T_{g}\right) .
$$

получаем следующее соотношение для логарифма относительной вязкости:

$$
\begin{gathered}
a_{T}=\eta(T) / \eta\left(T_{g}\right), \\
\ln a_{T}=-\left(\frac{1}{f_{g}}\right) \frac{T-T_{g}}{T-T_{g}+\left(f_{g} / \beta_{f}\right)},
\end{gathered}
$$

где $f_{g}$ - значение $f$ при $T=T_{g}, \beta_{f}-$ коэффициент теплового расширения флуктуационного объема $\left(\beta_{f}=(d f / d T)_{T=T_{g}}\right)$. Данное равенство представляет собой интерпретацию уравнения ВЛФ (Вильямса-Ландела-Ферри), получившего широкое распространение в физике аморфных полимеров [4] и стекол [5]. Эмпирические постоянные данного уравнения $C_{1}$ и $C_{2}$ выражаются через параметры модели делокализованных атомов следующим образом:

$$
C_{1}=\frac{1}{f_{g}} \quad \text { и } \quad C_{2}=\frac{f_{g}}{\beta_{f}} .
$$

Температурная зависимость вязкости стеклообразующих жидкостей успешно описывается известным уравнением ФФТ (Фогеля-Фульчера-Таммана)

$$
\eta=A \exp \left(\frac{B}{T-T_{0}}\right),
$$

которое практически эквивалентно соотношению ВЛФ (4) (см. ниже). Японские исследователи [6] показали применимость уравнения ФФТ к металлическим стеклам и определили для них значения эмпирических постоянных $A, B$ и $T_{0}$, а также $T_{g}$, которые приводятся в табл. 1. Представляет интерес установление их связи с параметрами модели делокализованных атомов.

С этой целью запишем данное уравнение при $T=T_{g}$ и с учетом (6) найдем логарифм отношения относительной вязкости $a_{T}=\eta(T) / \eta\left(T_{g}\right)$

$$
\ln a_{T}=-\left(\frac{B}{T_{g}-T_{0}}\right) \frac{T-T_{g}}{T-T_{0}} .
$$

Как видно, полученное выражение совпадает с уравнением ВЛФ (4), откуда имеем

$$
\frac{1}{f_{g}}=\frac{B}{T_{g}-T_{0}} \quad \text { и } \quad \frac{f_{g}}{\beta_{f}}=T_{g}-T_{0},
$$

что в свою очередь приводит к следующей связи параметров модели делокализованных атомов с постоянными уравнения ФФТ:

$$
\begin{gathered}
f_{g}=\frac{T_{g}-T_{0}}{B}, \\
\beta_{f}=\frac{1}{B} .
\end{gathered}
$$

\section{Результаты расчета и их обсуждение}

В табл. 1 приводятся значения параметров модели делокализованных атомов $f_{g}, \beta_{f}, \Delta \varepsilon_{e}-$ энергии делокализации атома [1]

$$
\Delta \varepsilon_{e}=R T_{g} \ln \left(1 / f_{g}\right)
$$

для металлических стекол, рассчитанные с помощью соотношений (7)-(9) на основе экспериментальных данных о постоянных уравнения ФФТ. Там же приведены значения параметров уравнения ВЛФ $C_{1}$ и $C_{2}$, определенные по формулам

$$
C_{1}=B /\left(T_{g}-T_{0}\right), \quad C_{2}=T_{g}-T_{0} .
$$

1. Прежде всего обратим внимание на то обстоятельство, что доля флуктуационного объема аморфных металлических сплавов $f_{g}$, замороженная при температуре стеклования (7), является практически постоянной величиной (табл. 1)

$$
f_{g}=\left(\frac{\Delta V_{e}}{V}\right)_{T=T_{g}} \approx \text { const } \approx 0.025-0.027,
$$

и, что примечательно, совпадает с данными о величине $f_{g}$ у аморфных полимеров и неорганических стекол (табл. 2) [1].

Это означает справедливость для металлических стекол того же критерия стеклования (10), что и для аморфных полимеров и неорганических стекол [1], и подтверждает известный факт: основные закономерности процесса перехода жидкость-стекло в качественном отношении оказываются универсальными для всех аморфных веществ независимо от их природы [7-11].

Полагаем, что делокализованные возбужденные атомы выше температуры стеклования $T_{g}$ обеспечивают вязкое течение стеклообразующих расплавов. Их подвижность и относительное число определяются долей флуктуационного объема $f=\left(\Delta V_{e} / V\right)$. При охлаждении расплава в области стеклования величина $f$ уменьшается до минимального критического значения $f_{g} \approx 2-3 \%$ (соотношение $(10)$ ), при котором достигается предельно 
Таблица 1. Постоянные уравнения ФФТ $\left(\eta_{0}, B, T_{0}\right)$ и параметры модели делокализованных атомов для металлических стекол (использованы данные [6])

\begin{tabular}{|c|c|c|c|c|c|c|c|c|c|c|c|c|}
\hline \multirow{2}{*}{ № } & \multirow{2}{*}{ Аморфный сплав } & \multirow{2}{*}{$T_{g}, \mathrm{~K}$} & \multirow{2}{*}{$A \cdot 10^{3} \mathrm{~Pa} \cdot \mathrm{s}$} & \multirow{2}{*}{$B, \mathrm{~K}$} & \multirow{2}{*}{$T_{0}, \mathrm{~K}$} & \multirow{2}{*}{$f_{g}$} & \multirow{2}{*}{$\beta_{f} \cdot 10^{4}, \mathrm{~K}^{-1}$} & $\Delta \varepsilon_{e}$ & $\Delta F_{\infty}$ & $\Delta F_{\eta g}$ & \multirow{2}{*}{$C_{1}, \mathrm{~K}$} & \multirow{2}{*}{$C_{2}, \mathrm{~K}$} \\
\hline & & & & & & & & \multicolumn{3}{|c|}{$\mathrm{kJ} / \mathrm{mol}$} & & \\
\hline 1 & $\mathrm{Ni}$ & 430 & 2.0 & 4700 & 295 & 0.029 & 2.1 & 13 & 39 & 124 & 35 & 135 \\
\hline 2 & $\mathrm{Ni}_{62.4} \mathrm{Nb}_{37.6}$ & 945 & 0.49 & 5380 & 810 & 0.025 & 1.9 & 29 & 43 & 313 & 40 & 135 \\
\hline 3 & $\mathrm{Ni}_{75} \mathrm{Si}_{8} \mathrm{~B}_{17}$ & 782 & 2.53 & 4280 & 670 & 0.026 & 2.3 & 24 & 36 & 248 & 38 & 112 \\
\hline 4 & $\mathrm{Fe}_{91} \mathrm{~B}_{9}$ & 600 & 14.1 & 4635 & 513 & 0.019 & 2.2 & 20 & 38 & 266 & 53 & 87 \\
\hline 5 & $\mathrm{Fe}_{89} \mathrm{~B}_{11}$ & 640 & 8.53 & 4625 & 515 & 0.027 & 2.2 & 19 & 38 & 197 & 37 & 125 \\
\hline 6 & $\mathrm{Fe}_{83} \mathrm{~B}_{17}$ & 760 & 3.3 & 4630 & 638 & 0.026 & 2.2 & 23 & 38 & 240 & 38 & 122 \\
\hline 7 & $\mathrm{Fe}_{41.5} \mathrm{Ni}_{41.5} \mathrm{~B}_{17}$ & 720 & 3.78 & 4500 & 601 & 0.026 & 2.2 & 22 & 37 & 226 & 38 & 119 \\
\hline 8 & $\mathrm{Fe}_{79} \mathrm{Si}_{10} \mathrm{~B}_{11}$ & 818 & 1.9 & 4505 & 701 & 0.026 & 2.2 & 25 & 37 & 262 & 38 & 117 \\
\hline 9 & $\mathrm{Fe}_{80} \mathrm{P}_{13} \mathrm{C}_{7}$ & 736 & 2.25 & 4600 & 616 & 0.026 & 2.2 & 22 & 38 & 234 & 38 & 120 \\
\hline 10 & $\mathrm{Pd}_{82} \mathrm{Si}_{18}$ & 657 & 6.32 & 3730 & 557 & 0.027 & 2.7 & 20 & 31 & 204 & 37 & 100 \\
\hline 11 & $\mathrm{Pd}_{77.5} \mathrm{Cu}_{6} \mathrm{Si}_{16.5}$ & 653 & 2.57 & 3820 & 553 & 0.026 & 2.6 & 20 & 32 & 207 & 38 & 100 \\
\hline 12 & $\mathrm{Pd}_{40} \mathrm{Ni}_{40} \mathrm{P}_{20}$ & 602 & 1.5 & 3600 & 509 & 0.026 & 2.8 & 18 & 30 & 194 & 39 & 93 \\
\hline 13 & $\mathrm{Pt}_{60} \mathrm{Ni}_{15} \mathrm{P}_{25}$ & 500 & 5.31 & 3560 & 405 & 0.027 & 2.8 & 15 & 30 & 156 & 37 & 95 \\
\hline 14 & $\mathrm{Te}$ & 290 & 0.13 & 3790 & 198 & 0.024 & 2.6 & 9 & 31 & 99 & 41 & 92 \\
\hline 15 & $\mathrm{Co}_{75} \mathrm{Si}_{15} \mathrm{~B}_{10}$ & 785 & 2.87 & 4190 & 675 & 0.26 & 2.4 & 24 & 35 & 248 & 38 & 110 \\
\hline 16 & $\mathrm{Ge}$ & 750 & 18.3 & 1930 & 700 & 0.026 & 5.2 & 23 & 16 & 241 & 39 & 50 \\
\hline
\end{tabular}

Примечание. $f_{g}=B /\left(T_{g}-T_{0}\right), \beta_{f}=1 / B, \Delta F_{\infty}=R B, \Delta \varepsilon_{e}=R T_{g} \ln \left(1 / f_{g}\right)$.

Таблица 2. Постоянные уравнения ВЛФ $C_{1}$ и $C_{2}$ и параметры модели делокализованных атомов для аморфных полимеров и неорганических стекол $[4,5]$

\begin{tabular}{|c|c|c|c|c|c|c|c|c|c|}
\hline \multirow{2}{*}{ Стекло } & \multirow{2}{*}{$T_{g}, \mathrm{~K}$} & \multirow{2}{*}{$C_{1}, \mathrm{~K}$} & \multirow{2}{*}{$C_{2}, \mathrm{~K}$} & \multirow{2}{*}{$f_{g}$} & $\beta \cdot 10^{4}$ & $\Delta \beta \cdot 10^{4}$ & $\Delta \varepsilon_{e}$ & $\Delta F_{\infty}$ & $\Delta F_{\eta g}$ \\
\hline & & & & & \multicolumn{2}{|c|}{$\mathrm{K}^{-1}$} & \multicolumn{3}{|c|}{$\mathrm{kJ} / \mathrm{mol}$} \\
\hline \multicolumn{10}{|l|}{$\begin{array}{c}\mathrm{Na}_{2} \mathrm{O}-\mathrm{SiO}_{2} \\
\mathrm{Na}_{2} \mathrm{O}, \mathrm{mol} . \%\end{array}$} \\
\hline 19 & 746 & 38 & 317 & 0.026 & 0.86 & - & 23 & 100 & 235 \\
\hline 32.9 & 704 & 36 & 275 & 0.028 & 1.03 & 0.86 & 21 & 83 & 210 \\
\hline 44.8 & 667 & 44 & 211 & 0.023 & 1.08 & 1.39 & 21 & 78 & 244 \\
\hline $\mathrm{Na}_{2} \mathrm{O}-\mathrm{PbO}-\mathrm{SiO}_{2}$ & 761 & 32 & 280 & 0.031 & 1.1 & 1.0 & 22 & 75 & 203 \\
\hline $\begin{array}{c}\mathrm{Na}_{2} \mathrm{O}-\mathrm{CaO}-\mathrm{SiO}_{2} \\
\mathrm{~K}_{2} \mathrm{O}-\mathrm{B}_{2} \mathrm{O}_{3} \\
\mathrm{~K}_{2} \mathrm{O}, \mathrm{mol} . \%\end{array}$ & 833 & 37 & 320 & 0.027 & 0.9 & 0.9 & 25 & 98 & 254 \\
\hline 8.5 & 623 & 30 & 89 & 0.030 & 2.6 & - & 18 & 32 & 173 \\
\hline 34.4 & 701 & 33 & 117 & 0.026 & 2.0 & - & 21 & 45 & 223 \\
\hline \multicolumn{10}{|l|}{$\mathrm{Na}_{2} \mathrm{O}-\mathrm{GeO}_{2}$} \\
\hline $\mathrm{Na}_{2} \mathrm{O}, \mathrm{mol} . \%$ & & & & & & & & & \\
\hline 5 & 729 & 40 & 220 & 0.025 & 1.1 & - & 23 & 73 & 242 \\
\hline 25 & 755 & 40 & 160 & 0.025 & 1.6 & - & 23 & 53 & 250 \\
\hline Поливинилацетат & 305 & 36 & 47 & 0.028 & 5.9 & 5.0 & 9 & 14 & 91 \\
\hline Натуральный каучук & 300 & 38 & 54 & 0.026 & 4.8 & 4.0 & 9 & 17 & 96 \\
\hline Метакрилатный этиловый полимер & 335 & 40 & 65 & 0.025 & 3.7 & 3.0 & 10 & 22 & 113 \\
\hline
\end{tabular}

При ие чан ие. $f_{g}=1 / C_{1}, \beta_{f}=1 / C_{1} C_{2}, \Delta \varepsilon_{e}=R T_{g} \ln \left(1 / f_{g}\right), \Delta F_{\infty}=R C_{1} C_{2}, \Delta F_{\eta g}=C_{1} R T_{g}, \Delta \beta-$ скачок коэффициента теплового расширения при $T=T_{g}$.

высокое значение вязкости $\eta\left(T_{g}\right) \approx 10^{12} \mathrm{~Pa} \cdot \mathrm{s}:$ прекращается вязкое течение и аморфное вещество переходит из жидкого в твердое стеклообразное состояние. В самом деле, подавляющее большинство аморфных веществ переходит в стеклообразное состояние в первом приближении при одной и той же вязкости [10]

$$
\eta_{g}=\eta\left(T_{g}\right) \approx \text { const } \approx 10^{12} \mathrm{~Pa} \cdot \mathrm{s} .
$$

По данным о предэкспоненциальном множителе $\eta_{0} \approx$ const $\approx 10^{-2.5} \mathrm{~Pa} \cdot \mathrm{s}$ и вязкости $\eta_{g} \approx 10^{12} \mathrm{~Pa} \cdot \mathrm{s} \quad \mathrm{c}$ 
помощью уравнения (3) можно оценить долю флуктуационного объема $f_{g}$, замороженную при температуре стеклования $T=T_{g}$,

$$
f_{g}=\left(\ln \frac{\eta_{g}}{\eta_{0}}\right)^{-1} \approx 0.03,
$$

что согласуется с оценкой (10).

Таким образом, с этой точки зрения стеклование жидкостей объясняется замораживанием подвижности делокализованных возбужденных кинетических единиц. При температуре стеклования замораживается процесс делокализации атома (переход частицы из основного в возбужденное состояние). В процессе размягчения стекла при нагревании постепенно размораживается процесс делокализации атома и количество делокализованных атомов возрастает от малых значений в замороженном состоянии до их концентрации, соответствующей жидкому состоянию.

2. Коэффициент теплового расширения флуктуационного объема (КТР) металлических стекол при температуре стеклования $\beta_{f} \approx 2 \cdot 10^{-4} \mathrm{~K}^{-1}$ (табл. 1) примерно совпадает с КТР неорганических стекол, но заметно ниже КТР аморфных полимеров (табл. 2).

У большинства аморфных органических полимеров и неорганических стекол величина $\beta_{f}$ совпадает со скачком коэффициента объемного теплового расширения $\Delta \beta$ при температуре стеклования $T=T_{g}$ (табл. 2)

$$
\Delta \beta=\beta_{l}-\beta_{g}
$$

где $\beta_{l}$ и $\beta_{g}$ - коэффициенты теплового расширения соответственно выше $\left(\beta_{l}\right)$ и ниже $\left(\beta_{g}\right)$ температуры стеклования.

Можно предположить, что полученные значения $\beta_{f}$ для металлических стекол также совпадают со скачком их коэффициента теплового расширения $\Delta \beta$ при температуре стеклования.

3. Энергия делокализации атома в металлических стеклах $\Delta \varepsilon_{e} \approx 20-25 \mathrm{~kJ} / \mathrm{mol}$ (табл. 1) примерно совпадает с данными для неорганических стекол $\Delta \varepsilon_{e} \approx$ $\approx 20-23 \mathrm{~kJ} / \mathrm{mol}$ (табл. 2). Исключение составляет теллур, у которого величина $\Delta \varepsilon_{e}=9 \mathrm{~kJ} / \mathrm{mol}$ совпадает с энергией делокализации атома в аморфных полимерах $\Delta \varepsilon_{e}=9-10 \mathrm{~kJ} / \mathrm{mol}$. У последних энергия $\Delta \varepsilon_{e}$ близка к энергии межмолекулярных (ван-дер-ваальсовых) связей, a у оксидных неорганических стекол она связана с работой смещения мостикового атома кислорода в мостиках валентных связей $\mathrm{Si}-\mathrm{O}-\mathrm{Si}, \mathrm{Ge}-\mathrm{O}-\mathrm{Ge}, \mathrm{B}-\mathrm{O}-\mathrm{B}$ и т.д. $[1,12]$. Энергия $\Delta \varepsilon_{e}$ существенно ниже энергий активации вязкого течения и диффузионных процессов. Поэтому делокализация атома в аморфных веществах, в том числе в металлических стеклах, относится к низкоэнергетическим мелкомасштабным процессам $[1,2,12]$.

4. Из сравнения известного теоретического уравнения Эйринга (см. [5])

$$
\eta=\eta_{0} \exp \left[\frac{\Delta F_{\eta}(T)}{R T}\right]
$$

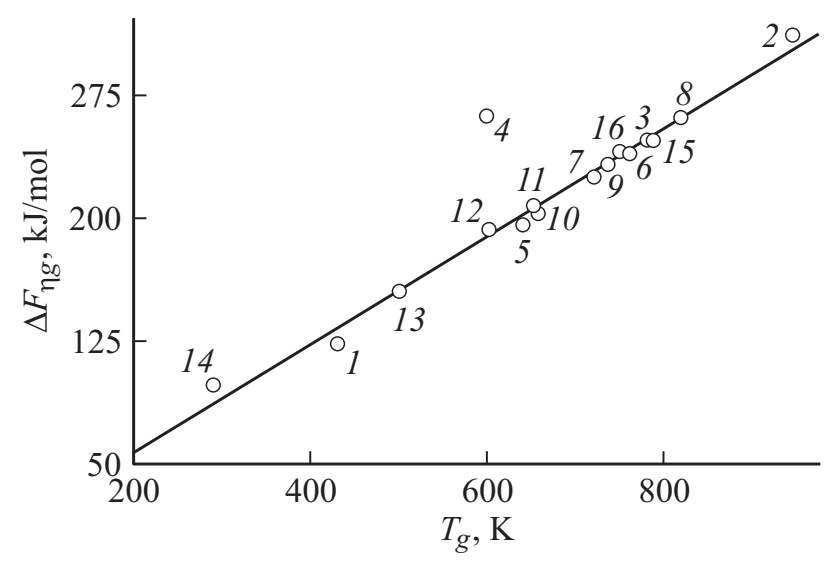

Корреляция между температурой стеклования $T_{g}$ и свободной энергией активации вязкого течения $\Delta F_{\eta g}$ при $T_{g}$ для металлических стекол. Номера точек соответствуют номерам стекол в табл. 1.

с соотношением ФФТ (6) в приближении $\eta_{0} \approx A$ получаем следующую температурную зависимость свободной энергии активации вязкого течения $\Delta F_{\eta}(T)$ :

$$
\Delta F_{\eta}=\frac{\mathrm{BRT}}{T-T_{0}}=\mathrm{RB}\left(1-\frac{T_{0}}{T}\right)^{-1},
$$

где произведение RB имеет смысл высокотемпературного предела $\left(T \gg T_{0}\right)$ свободной энергии активации текучести $\Delta F_{\infty}=\delta F_{\eta}(T \rightarrow \infty)$

$$
\Delta F_{\infty}=\mathrm{RB} .
$$

Из равенства (11) можно получить формулу для расчета величины $\Delta F_{\eta g}=\Delta F\left(T_{g}\right)$ при температуре стеклования

$$
\Delta F_{\eta g}=\frac{B}{\left(T_{g}-T_{0}\right)} \mathrm{RT}_{g} .
$$

Значения $\Delta F_{\infty}$ и $\Delta F_{\eta g}$, рассчитанные по формулам (12) и (13), приведены в табл. 1. Они по порядку величины близки к данным для неорганических стекол (табл. 2).

Принимая во внимание (7) и постоянство $f_{g} \cong$ const (табл. 1 и 2), приходим к заключению, что свободная энергия активации вязкого течения $\Delta F_{\eta g}$ пропорциональна температуре стеклования:

$$
\Delta F_{\eta g}=\left(1 / f_{g}\right) R T_{g}
$$

В самом деле, у металлических стекол между $\Delta F_{\eta g}$ и $T_{g}$ наблюдается линейная корреляция (рисунок).

\section{Заключение}

Доля флуктуационного объема $f_{g}$, замороженная при температуре стеклования $T_{g}$, у аморфных металлических сплавов и аморфных металлов (металлических стекол) оказывается практически постоянной величиной и составляет около $f_{g} \approx 0.026$, что совпадает с данными 
для аморфных полимеров и стекол и указывает на применимость условия стеклования $f_{g} \approx$ const $\approx 0.025$ к этим стеклообразным системам.

Энергия делокализации атома в металлических стеклах $(\Delta \varepsilon=20-25 \mathrm{~kJ} / \mathrm{mol})$ примерно такая же, как и у силикатных стекол. Еще один параметр модели делокализованных атомов - коэффициент теплового расширения флуктуационного объема $\beta_{f} \approx 2 \cdot 10^{-4} \mathrm{~K}^{-1}-$ у металлических стекол практически совпадает с данными для неорганических стекол.

У исследованных металлических стекол между температурой стеклования $T_{g}$ и свободной энергией активации вязкого течения $\Delta F_{\eta g}$ при $T_{g}$ наблюдается линейная корреляция.

Работа выполнена при финансовой поддержке Минобрнауки РФ (Грант № 1932).

\section{Список литературы}

[1] Сандитов Д.С. // ЖЭТФ. 2012. Т. 142. Вып. 1. С. 123-137.

[2] Сандитов Д.С., Мункуева С.Б., Булыгина Е.А. // ЖТФ. 2011. Т. 81. Вып. 10. С. 40-45.

[3] Соловьев А.Н., Каплун А.Б. Вибрационный метод измерения вязкости жидкостей. Новосибирск: Наука, 1970. 113 с.

[4] Ферри Дж. Вязкоупругие свойства полимеров. М.: ИЛ, 1963. $535 \mathrm{c}$.

[5] Сандитов Д.С., Бартенев Г.М. Физические свойства неупорядоченных структур. Новосибирск: Наука, 1982. $269 \mathrm{c}$.

[6] Судзуки К., Фузимора Х., Хасимото $К$. Аморфные металлы. М.: Металлургия, 1987. 328 с.

[7] Кобеко П.П. Аморфные вещества. М.; Л.: Изд-во AH CCCP, 1952. $431 \mathrm{c}$.

[8] Ростиашвили В.Г., Иржсак В.И., Розенберг Б.А. Стеклование полимеров. Л.: Химия, 1987. 192 с.

[9] Мазурин О.В. Стеклование. Л.: Наука, 1986. 210 с.

[10] Ojovan M.I. // Adv. Condens. Matter Phys., Article ID817829.2008.

[11] Тропин Т.В., Шмельцер Ю.В., Аксенов В.Л. // УФН. 2016. T. 186. № 1. C. 47-73.

[12] Сандитов Д.С., Бадмаев С.С. // ФХС. 2015. Т. 40. № 5. C. $621-630$. 